\title{
Higher diurnal salivary cortisol levels are related to smaller prefrontal cortex surface area in elderly men and women
}

\author{
Andreas Stomby ${ }^{1,2}$, Carl-Johan Boraxbekk ${ }^{3,4}$, Anders Lundquist ${ }^{4}$, Annelie Nordin ${ }^{5}$, \\ Lars-Göran Nilsson ${ }^{4,6}$, Rolf Adolfsson ${ }^{5}$, Lars Nyberg ${ }^{4,7,8}$ and Tommy Olsson' \\ 'Department of Public Health and Clinical Medicine, Medicine, Umeå University, Umeå, Sweden, \\ ${ }^{2}$ Region Jönköping County, Jönköping, Sweden, ${ }^{3}$ CEDAR, Center for Demographic and Aging Research, \\ ${ }^{4}$ Umeå Center for Functional Brain Imaging (UFBI), ${ }^{5}$ Department of Clinical Sciences, Psychiatry, \\ Umeå University, Umeå, Sweden, ${ }^{6}$ Department of Psychology, Stockholm University, Stockholm, \\ Sweden, ${ }^{7}$ Integrative Medical Biology, Physiology, and ${ }^{8}$ Radiation Sciences, Diagnostic Radiology, \\ Umeå University, Umeå, Sweden
}

Correspondence should be addressed to A Stomby Email

andreas.stomby@umu.se

\begin{abstract}
Objective: Elevated cortisol levels with aging have been associated with atrophy of the hippocampus and prefrontal cortex (PFC), as well as with impaired cognitive functions in men. However, coexisting diseases have confounded many studies examining these relationships. Studies in women are lacking. Our objective was to test whether salivary cortisol levels were related to morphology of the hippocampus and the PFC, and to cognitive performance.

Design: A cross-sectional study including 200 elderly (55-80 years old) men and women.

Method: We used magnetic resonance imaging, tests of episodic-, semantic-, and working memory, visuospatial ability, and cortisol levels in four saliva samples collected during 1 day.

Results: Area under the curve (AUC) for cortisol levels was negatively related to cortical surface area of the left anterior cingulate gyrus (caudal $P<0.001$; rostral $P=0.006$ ), right lateral orbitofrontal cortex $(P=0.004)$, and right rostral middle frontal gyrus $(P=0.003)$. In women, there was also a negative relationship with cortical surface area in the left rostral middle frontal gyrus $(P=0.006)$. No relationship was found between cortisol levels and hippocampal volume.

Conclusion: This study suggests that the structure of the medial PFC is related to cortisol levels in both elderly women
\end{abstract} and men.

European Journal of

Endocrinology

(2016) 175, 117-126

\section{Introduction}

The hypothalamic-pituitary-adrenal axis (HPAA) regulates cortisol secretion from the adrenal glands. The HPAA has a clear circadian rhythm with high cortisol levels in the morning increasing rapidly after awakening and then decreasing throughout the day to reach a nadir during the late evening. It is also activated in response to threats to homeostasis (stress) (1). Following release, cortisol negatively feedback upon the HPAA, acting via glucocorticoid receptors (GR) and mineralocorticoid
() 2016 European Society of Endocrinology Printed in Great Britain receptors (MR). Negative feedback occurs not only in the hypothalamus and pituitary but also in higher brain structures, notably the prefrontal cortex (PFC) and the hippocampus (HC). The highest receptor density is present in the HC (2). MRs have high affinity for cortisol and are, therefore, activated during the nadir at which cortisol levels are low, while the low-affinity GR requires higher cortisol levels achieved at the diurnal peak or on stress to become activated $(3,4)$. In addition to regulation of 
the HPAA, the HC is central to episodic, declarative, and spatial memory function, and the PFC mediates executive function resulting in goal-directed behaviors (5). Thus, the $\mathrm{HC}$ and PFC can integrate the cognitive resources and the neuroendocrine response needed to cope with a stressor.

The relationship between cortisol and these brain regions is bidirectional (6). Several studies have found structural alterations of neurons such as shortening of apical dendrites, decreased dendritic arborization, and a decreased number of dendritic spines on pyramidal neurons in the $\mathrm{HC}$ and medial PFC in response to increased glucocorticoid levels and stress $(7,8)$. It has been proposed that circulating cortisol levels are associated with cognitive performance in healthy humans, including both HC-dependent functions $(9,10)$ and PFC-dependent functions $(10,11,12)$. The relationships appear stronger with aging, and increased glucocorticoid action may underpin some of the cognitive loss in a subset of aged individuals.

In addition, some $(9,13,14)$ but not all $(15,16)$ studies have found a negative association between cortisol levels in plasma or saliva and HC volume. High salivary cortisol levels have also been related to a thinner cortex in several PFC regions in healthy men (15), but this has not been tested in women. Importantly, experimental research suggests gender-specific differences in the effects of glucocorticoids on PFC and HC neurons $(17,18)$. Furthermore, a putative association between cortisol levels and HC volume is confounded by small samples (9), coexisting depression (13) or cardiovascular disease (14). Thus, the generalizability of previous results remains unclear.

Our hypothesis was that salivary cortisol levels are negatively associated with volumes of the PFC and hippocampus. We used both cortical area and thickness measurements for the PFC, as it has been suggested that these estimates may relate differently to cortisol levels (15). We also hypothesized that salivary cortisol levels would be negatively related to cognitive functions that require a high level of PFC and HC functionality. We used a population-based sample of middle-aged and elderly women and men to evaluate putative gender differences in these associations.

\section{Subjects and methods}

\section{Participants}

All participants were part of the ongoing Betula Prospective Cohort Study on memory, health, and aging (19). In this study, we used data collected at 20082010. Participants underwent a health examination consisting of anthropometric measurements, bloodpressure measurement, and questions regarding drug use and medical history over the last 5 years. Dementia was investigated via routine clinical methods. In a subsequent visit, cognitive tests were completed. The mean time between the health examination and cognitive testing was 12 days (s.D. 12.6 days, range 3-91 days). Additionally, 292 participants with complete cognitive data were randomly invited to take part in magnetic resonance imaging of the brain (MRI) after stratification for gender and age until a predefined number of MRI examinations were completed. Of the 292 individuals who participated in the MRI study, 92 participants were excluded due to the following reasons: incomplete saliva sampling ( $<4$ samples, $n=87$ ), diagnosed with dementia $(n=3)$, glucocorticoid drug treatment $(n=7)$, and inaccurate MRI image processing $(n=1)$ leaving 200 subjects for the analysis. For these 200 subjects, the mean time between cognitive testing and MRI examination was 298 days (s.D. 90 days, range 88-524 days).

The cohort consisted of 100 men and 100 women aged 55-80 years; there were no significant gender-based differences in age, level of education, or Mini-Mental State Examination (MMSE) results (Table 1). Six participants had mild cognitive impairment (20). Forty percent of the participants were normal weight (BMI $18.5-25 \mathrm{~kg} / \mathrm{m}^{2}$ ), $47 \%$ were overweight (BMI $25-30 \mathrm{~kg} / \mathrm{m}^{2}$ ), and $13 \%$ were obese (BMI $\geq 30 \mathrm{~kg} / \mathrm{m}^{2}$ ). Sixty-seven percent of the sample had hypertension (blood pressure $>140 / 90 \mathrm{mmHg}$, antihypertensive drug treatment, or self-reported diagnosis of hypertension, Table 1). Overall, 9.5\% of patients had heart disease (angina pectoris or previous myocardial infarction), $1 \%$ had a history of stroke, and $7 \%$ had type 2 diabetes. Eight participants were current smokers. Fifteen participants used antidepressive drugs, one used antipsychotic drugs, and three used antiepileptic drugs. Fourteen participants had sought care for depression, exhaustion, or memory complaints during the 5 years preceding the measurements, and one suffered from Parkinson's disease. The 92 participants who did not provide four saliva samples were treated with glucocorticoids or who had dementia were excluded from this study

Table 1 Basic characteristics. Data are presented as mean (s.D.).

\begin{tabular}{|c|c|c|c|}
\hline & Men & Women & $\boldsymbol{P}$ \\
\hline Total number of participants $(n)$ & 100 & 100 & \\
\hline Age (years) & $67(8)$ & $67(8)$ & 0.92 \\
\hline Level of education (years) & $12(4)$ & $12(4)$ & 0.41 \\
\hline MMSE score & $28(2)$ & $28(2)$ & 0.82 \\
\hline
\end{tabular}

$P=P$-value for differences between men and women tested with independent samples $t$-test. 
and did not differ with regards to gender distribution, age, level of education, body mass index, hypertension, or MMSE score compared with the 200 participants included in the final analysis. The study was conducted in accordance with the Declaration of Helsinki, and all participants provided written informed consent before study inclusion.

\section{Saliva cortisol levels}

At time point 5 (during a workday or weekend), participants were instructed to sample saliva during 1 day at 07:00, 11:00, 16:00, and 23:00 h. All but two participants sampled saliva during the period between the health examination and cognitive testing. The two participants that did not return their samples at the time of cognitive testing did so 24 and 59 days later. Participants were instructed not to eat, brush their teeth, or smoke $1 \mathrm{~h}$ before sampling and to store their saliva in the refrigerator for up to 1 day before handing it over to the research staff. Saliva samples were frozen at $-20^{\circ} \mathrm{C}$ in $4-6$ years until cortisol levels were measured. Samples were analyzed in one batch with a chemiluminescence immunoassay (IBL International $\mathrm{GmbH}$, Hamburg, Germany). The inter- and intra-assay coefficients of variation for the analysis were $<8 \%$. The AUC with respect to ground was calculated according to the formula presented by Pruessner and coworkers (21).

\section{MRI acquisition}

Brain imaging was performed on a General Electric $3 \mathrm{~T}$ Discovery MR750 scanner with a 32-channel head coil. High-resolution T1-weighted images were collected with a 3D fast spoiled gradient echo sequence using the following parameters: 180 slices with $1 \mathrm{~mm}$ thickness, TR $8.2 \mathrm{~ms}$, TE $3.2 \mathrm{~ms}$, flip angle $12^{\circ}$, field of view $25 \times 25 \mathrm{~cm}$.

\section{MRI data analysis}

FreeSurfer version 5.3 was used to anatomically segment the structural T1-weighted images. These methods were described previously in detail $(22,23)$. In short, images were normalized and motion-corrected, non-brain tissue was removed, and the images were transformed to the Talairach space. The maximum shift in signal intensity was used to localize the boundaries between gray matter, white matter, and cerebrospinal fluid. The white-matter surface was inflated to visualize the gyral and sulcal areas simultaneously. White matter, subcortical gray-matter structures, and the cortical surface were segmented. The
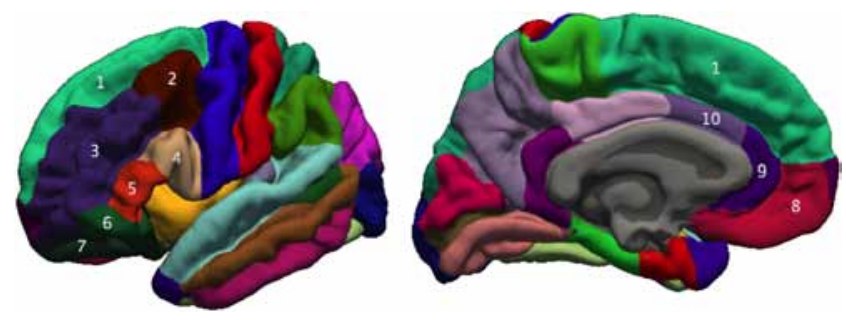

\section{Figure 1}

Cortical parcellation generated by Freesurfer according to the parcellation system by Desikan and coworkers (23). The association between salivary cortisol measures and the numbered regions in the prefrontal cortex was analyzed separately for the left and right hemispheres. Those areas that were significantly $(P<0.05$ corrected for the false discovery rate) associated with AUC cortisol levels in either hemispheres are given in italics. 1, superior frontal gyrus; 2, caudal middle frontal gyrus; 3, rostral middle frontal gyrus; 4, inferior frontal gyrus pars opercularis; 5 , inferior frontal gyrus pars triangularis; 6, inferior frontal gyrus pars orbitalis; 7, lateral orbitofrontal cortex; 8 , medial orbitofrontal cortex; 9 , rostral anterior cingulate gyrus; 10, caudal anterior cingulate gyrus.

distance from the border between white and gray matter to the pial surface was estimated with an automated algorithm and used as a measure of cortical thickness. Surface area was calculated at the border between white and gray matter. Segmentation of HC was performed automatically in Freesurfer according to a previously described method (22) and intracranial volume was estimated as specified previously (24). The MRI images were quality controlled by a technician and the data were checked for outliers. One participant was excluded due to inadequate imaging processing.

The association with saliva cortisol levels was evaluated for the following brain areas: superior, middle, and inferior frontal gyrus; the anterior cingulate gyrus; orbitofrontal cortices (Fig. 1); and the HC. Each hemisphere was analyzed separately since previous research has suggested that regulation of the HPAA may be lateralized (25).

\section{Cognitive testing}

The following five episodic memory tests were used as a composite measure of episodic memory performance: immediate free recall of nouns in 16 short verb-noun sentences encoded verbally and visually; delayed cued recall of nouns in the same sentences; immediate free recall of nouns in 16 other short verb-noun sentences encoded by enactment; delayed cued recall of nouns in the same 
sentences; and immediate free recall of 12 nouns encoded verbally. Three tests were used as a composite measure of semantic memory performance. For $1 \mathrm{~min}$, participants generated as many words as possible: (1) starting with an A, (2) five-letter words beginning with $\mathrm{M}$, and (3) professions starting with a B. Adding up the score from each individual test created both composite measures. Visuospatial ability was tested with the block-design task from the Wechsler Adult Intelligence Scale. A 2-back test of 20 words was used to assess working memory performance; results were reported as the percentage of correct answers.

\section{Statistical analysis}

The AUC for cortisol levels and the cortisol level at 23:00 h had skewed distributions and were logarithmically transformed before analysis. Multivariable linear regression analyses were performed with the hippocampal volume, cortical surface area, and cortical thickness of each analyzed brain region as dependent variables and saliva cortisol levels (AUC or cortisol level at 23:00 h) as predictors. These models included age, gender, and total intracranial volume as covariates. The following other potential confounders were also assessed but were not significantly associated with cortisol levels, cognitive performance, or brain structure, and were, therefore, excluded: body mass index, waist circumference, systolic blood pressure, reported diagnosis of hypertension or use of antihypertensive agents, type 2 diabetes, previous or present ischemic heart disease (angina or myocardial infarction), and stroke or transient ischemic attack. Total intracranial volume was not associated with cortical thickness and, therefore, excluded as a covariate in the models assessing cortical thickness. Subsequently, an interaction term with AUC for cortisol levels or cortisol level at 23:00 $\mathrm{h} \times$ gender was added to the model to evaluate putative gender effects on the relationship between cortisol levels and brain morphology. For those brain areas with a significant interaction $(P<0.05)$, post hoc analyses with men and women separated were performed.

Similar linear regression analyses were performed to test whether AUC for cortisol levels or cortisol level at 23:00 h was associated with performance on cognitive tests. Age, gender, and level of education and the interaction term cortisol measure $\mathrm{x}$ gender were added as covariates in the multivariable analysis because none of the other covariates were related to cognitive performance. Finally, we tested whether the cortical surface area and thickness of studied brain regions significantly associated with cortisol levels after adjustment for multiple comparisons were associated with performance on cognitive tests. In this multivariable analysis, we used age, gender, total intracranial volume, and level of education as covariates. All analyses were repeated, excluding the 37 participants with a reported history of epilepsy, Parkinson's disease, depression, schizophrenia, memory complaints, and/or exhaustion syndrome.

A two-tailed alpha level $<0.05$ was considered to be significant. The false discovery rate (FDR) (26) corrected for 20 tests at an alpha-level $<0.05$ was used to test whether the results remained significant after adjustment for multiple comparisons.

\section{Results}

\section{Cortisol levels and prefrontal cortical surface area}

Cortisol levels were highest in the morning, decreased throughout the day, and were lowest at 23:00 h, as expected (Table 2). No associations were found between cortisol levels and gender, age, or level of education (data not shown).

In general, women had smaller hippocampi, smaller cortical surface areas, and greater cortical thickness compared with men (Supplementary Table 1, see section on supplementary data given at the end of this article). After adjusting for covariates, the AUC for cortisol levels was significantly associated with smaller cortical surface area in eight prefrontal brain regions; left rostral and caudal anterior cingulate gyrus; right lateral orbitofrontal cortex; left and right medial orbitofrontal cortex; left and right rostral middle frontal gyrus; and right superior frontal gyrus (Table 3). The association in the medial orbitofrontal cortices and superior frontal gyrus did not reach an FDR adjusted $P<0.05$. The strongest association was with surface area of the left caudal anterior cingulate cortex (Fig. 2).

The interaction between AUC for cortisol levels and gender was nonsignificant in all brain regions except the left rostral middle frontal gyrus, indicating that these

Table 2 Cortisol levels in saliva. Data are presented as median (interquartile range).

\begin{tabular}{|c|c|c|c|}
\hline \multirow[b]{2}{*}{ Time point $(\mathrm{h})$} & \multicolumn{2}{|c|}{ Median cortisol level (nmol/L) } & \multirow[b]{2}{*}{$P$} \\
\hline & Men $(n=100)$ & Women $(n=100)$ & \\
\hline 07:00 & $13.6(9.1)$ & $13.8(10.4)$ & 0.18 \\
\hline $11: 00$ & $5.9(4.8)$ & $5.4(6.0)$ & 0.46 \\
\hline $16: 00$ & $3.3(3.0)$ & $3.4(2.8)$ & 0.75 \\
\hline 23:00 & $1.7(1.6)$ & $2.0(1.7)$ & 0.29 \\
\hline AUC for all time points & $84.1(48.0)$ & $85.1(56.2)$ & 0.27 \\
\hline
\end{tabular}

AUC, area under the curve given as $(\mathrm{nmol} / \mathrm{L} \times \mathrm{h}) . P=P$-value for differences between men and women tested with Mann-Whitney $U$ test. 
Table 3 Associations between area under the curve (AUC) for cortisol levels in saliva and cortical surface area and thickness in prefrontal brain regions. Two models were tested: A included age, gender, and estimated intracranial volume as covariates; $B$ included the same covariates plus the interaction term AUC cortisol $\times$ gender. Cortical thickness models did not include estimated intracranial volume. For the brain, regions with a significant AUC cortisol $\times$ gender interaction results were presented from model B. Results given in bold italics were significant after correction for the false discovery rate (FDR) with a $P<0.05$. The results that did not remain significant after FDR correction are given in italics.

\begin{tabular}{|c|c|c|c|c|}
\hline \multirow[b]{2}{*}{ Brain region } & \multicolumn{2}{|c|}{ AUC cortisol } & \multicolumn{2}{|c|}{ AUC cortisol $\times$ gender } \\
\hline & $\beta$ & $P$ & $\beta$ & $P$ \\
\hline \multicolumn{5}{|l|}{ Cortical surface area } \\
\hline L Rostral anterior cingulate gyrus & -0.17 & 0.006 & 0.077 & 0.40 \\
\hline R Rostral anterior cingulate gyrus & -0.068 & 0.29 & 0.01 & 0.91 \\
\hline L Caudal anterior cingulate gyrus & -0.29 & 0.000021 & -0.004 & 0.97 \\
\hline $\mathrm{R}$ Caudal anterior cingulate gyrus & 0.053 & 0.44 & 0.083 & 0.40 \\
\hline L Lateral orbitofrontal cortex & -0.089 & 0.14 & -0.064 & 0.47 \\
\hline R Lateral orbitofrontal cortex & -0.17 & 0.004 & -0.094 & 0.29 \\
\hline L Medial orbitofrontal cortex & -0.12 & 0.042 & -0.024 & 0.79 \\
\hline R Medial orbitofrontal cortex & -0.15 & 0.018 & -0.032 & 0.73 \\
\hline L Rostral middle frontal gyrus & -0.22 & 0.006 & 0.20 & 0.014 \\
\hline R Rostral Middle frontal gyrus & -0.16 & 0.003 & 0.12 & 0.13 \\
\hline L Caudal middle frontal gyrus & -0.08 & 0.20 & 0.10 & 0.26 \\
\hline R Caudal middle frontal gyrus & -0.089 & 0.16 & -0.041 & 0.66 \\
\hline L Superior frontal gyrus & -0.085 & 0.12 & 0.062 & 0.43 \\
\hline$R$ Superior frontal gyrus & -0.11 & 0.049 & 0.10 & 0.21 \\
\hline L Inferior frontal gyrus pars orbitalis & 0.01 & 0.87 & 0.092 & 0.31 \\
\hline L Inferior frontal gyrus pars triangularis & 0.032 & 0.63 & 0.076 & 0.43 \\
\hline L Inferior frontal gyrus pars opercularis & 0.055 & 0.40 & -0.036 & 0.71 \\
\hline $\mathrm{R}$ Inferior frontal gyrus pars orbitalis & -0.089 & 0.16 & -0.046 & 0.62 \\
\hline $\mathrm{R}$ Inferior frontal gyrus pars triangularis & -0.02 & 0.76 & 0.071 & 0.46 \\
\hline $\mathrm{R}$ Inferior frontal gyrus pars opercularis & -0.007 & 0.92 & 0.083 & 0.38 \\
\hline \multicolumn{5}{|l|}{ Cortical thickness } \\
\hline L Rostral anterior cingulate gyrus & 0.14 & 0.036 & 0.004 & 0.97 \\
\hline R Rostral anterior cingulate gyrus & -0.014 & 0.84 & -0.09 & 0.39 \\
\hline L Caudal anterior cingulate gyrus & 0.055 & 0.44 & 0.041 & 0.69 \\
\hline R Caudal anterior cingulate gyrus & 0.05 & 0.48 & -0.18 & 0.084 \\
\hline L Lateral orbitofrontal cortex & 0.063 & 0.37 & 0.029 & 0.78 \\
\hline R Lateral orbitofrontal cortex & 0.096 & 0.17 & 0.019 & 0.85 \\
\hline L Medial orbitofrontal cortex & 0.16 & 0.029 & -0.054 & 0.60 \\
\hline R Medial orbitofrontal cortex & 0.12 & 0.081 & 0.055 & 0.59 \\
\hline L Rostral middle frontal gyrus & -0.021 & 0.74 & -0.14 & 0.15 \\
\hline R Rostral Middle frontal gyrus & 0.021 & 0.75 & -0.12 & 0.23 \\
\hline L Caudal middle frontal gyrus & 0.21 & 0.035 & -0.20 & 0.041 \\
\hline$R$ Caudal middle frontal gyrus & 0.23 & 0.02 & -0.27 & 0.006 \\
\hline L Superior frontal gyrus & 0.10 & 0.13 & -0.16 & 0.11 \\
\hline R Superior frontal gyrus & 0.043 & 0.52 & -0.13 & 0.19 \\
\hline L Inferior frontal gyrus pars orbitalis & 0.002 & 0.97 & -0.035 & 0.72 \\
\hline L Inferior frontal gyrus pars triangularis & -0.09 & 0.20 & -0.091 & 0.37 \\
\hline L Inferior frontal gyrus pars opercularis & 0.059 & 0.41 & -0.16 & 0.12 \\
\hline $\mathrm{R}$ Inferior frontal gyrus pars orbitalis & 0.11 & 0.11 & -0.016 & 0.87 \\
\hline $\mathrm{R}$ Inferior frontal gyrus pars triangularis & -0.001 & 0.98 & -0.17 & 0.091 \\
\hline$R$ Inferior frontal gyrus pars opercularis & -0.008 & 0.91 & -0.24 & 0.023 \\
\hline
\end{tabular}

$\beta$, standardized $\beta$-cofficient; $P, P$-value; $L$, left; $R$, right.

negative associations were present in both men and women (Table 3). In the left rostral middle frontal gyrus, the negative association between AUC for cortisol levels and cortical surface area was present in women $(\beta=-0.28$, $P=0.003)$ but not in men $(\beta=0.069, P=0.44)$.

\section{Cortisol levels and prefrontal cortical thickness}

AUC for cortisol levels was positively associated with cortical thickness in the left rostral anterior cingulate gyrus, left medial orbitofrontal cortex, and the caudal 


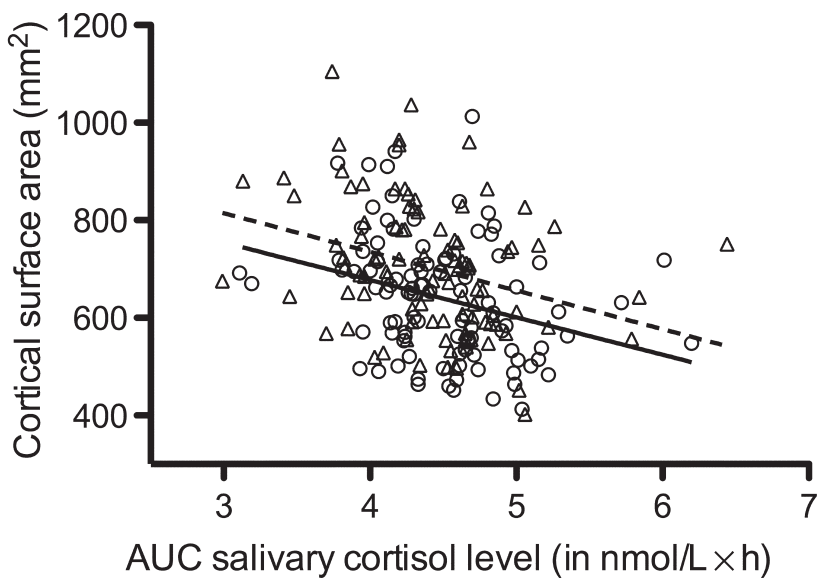

Figure 2

The relationship between cortical surface area in the left caudal anterior cingulate gyrus and area under the curve (AUC) for saliva cortisol levels measured four times during 1 day. Pearson correlation coefficient: for women, $r=-0.301$, $P=0.002$ (continuous line); for men, $r=-0.314, P=0.001$ (dashed line). Circles, women; triangles, men.

middle frontal gyrus bilaterally (Table 3), but none of these associations reached an FDR adjusted $P<0.05$. In the left and right caudal middle frontal gyrus, there was a significant interaction between AUC for cortisol levels and gender (Table 3), and the post hoc analysis revealed that the positive association was present in women (left $\beta=0.22, P=0.03$; right $\beta=0.26, P=0.009$ ) but not in men (left $\beta=-0.054, P=0.56$; right $\beta=-0.12, P=0.19$ ).

All associations between cortisol levels and cortical surface area and thickness remained significant when the 37 participants with a reported history of epilepsy, Parkinson's disease, depression, schizophrenia, memory complaints, exhaustion syndrome, and/or drug treatments related to these disorders were excluded from the analyses (data not shown).

\section{Cortisol levels and hippocampal volume}

Total HC volume was not associated with either AUC for cortisol levels (left: $\beta=-0.028, P=0.64$, right: $\beta=-0.018$, $P=0.77$ ) or cortisol level at 23:00h (left: $\beta=-0.028$, $P=0.63$, right: $\beta=0.009, P=0.89$ ).

\section{Cortisol levels and cognitive test results}

Women performed better than men on the episodic and semantic memory tasks (Table 4). There were no significant associations between the cortisol measures and
Table 4 Cognitive test results. Data are presented as mean (s.D.).

\begin{tabular}{l}
\hline Cognitive test results \\
\hline Episodic memory composite \\
( $n$ remembered items) \\
Semantic memory composite \\
( $n$ generated words) \\
Block-design task (score) \\
Two-back test (percent \\
correct answers)
\end{tabular}

\begin{tabular}{|c|c|c|}
\hline Men & Women & $\boldsymbol{P}$ \\
\hline $37.4(8.8)$ & $41.6(8.9)$ & 0.001 \\
\hline $30.3(9.7)$ & 34.6 (10.4) & 0.012 \\
\hline $29.4(8.4)$ & $27.9(9.5)$ & 0.27 \\
\hline $73 \%(20)$ & $74 \%(20)$ & 0.85 \\
\hline
\end{tabular}

$P$, difference between men and women tested with an independent samples $t$-test.

cognitive test performance (Table 5). However, there was a significant interaction between AUC for cortisol levels and gender on performance on the two-back test (Table 5). The post hoc analysis revealed that in men, higher AUC cortisol levels were associated with lower test scores $(\beta=-0.21, P=0.044)$, but this association was not present in women $(\beta=0.14, P=0.18)$. Notably, this association did not remain significant after FDR adjustment and was driven by the two men with highest cortisol levels; when these were excluded from the model, the association disappeared $(\beta=-0.078, P=0.47)$.

\section{Prefrontal cortical surface area and cognitive performance}

Since higher AUC for cortisol levels were associated with smaller cortical surface areas in prefrontal brain regions after adjustment for multiple comparisons, the relationship between cortical surface area in these brain regions and cognitive test scores was analyzed. Cortical surface area in the left rostral anterior cingulate gyrus was associated with semantic memory performance $(\beta=0.18$, $P=0.022$ ) and cortical surface area in the left caudal anterior cingulate gyrus $(\beta=0.14, P=0.033)$ and right rostral middle frontal gyrus $(\beta=0.19, P=0.019)$ was associated with visuospatial performance. These associations did not remain significant after FDR adjustment.

\section{Discussion}

The AUC for saliva cortisol levels was associated with smaller cortical surface areas in the left anterior cingulate gyrus, the right lateral orbitofrontal cortex, and the rostral middle frontal gyrus bilaterally, i.e. subregions of the prefrontal cortex. Notably, these associations remained significant after adjusting for multiple comparisons. However, neither the AUC nor cortisol level at 23:00 h was related to $\mathrm{HC}$ volume. This pinpoints a link between 
Table 5 Associations between area under the curve (AUC) for cortisol levels, cortisol level at 23:00 h, and cognitive test results.

\begin{tabular}{|c|c|c|c|c|c|c|c|c|}
\hline \multirow[b]{2}{*}{ Cognitive test } & \multicolumn{2}{|c|}{ AUC cortisol } & \multicolumn{2}{|c|}{ AUC cortisol $\times$ gender } & \multicolumn{2}{|c|}{ Cortisol 23:00 h } & \multicolumn{2}{|c|}{ Cortisol 23:00 $\mathrm{h} \times$ gender } \\
\hline & $\beta$ & $P$ & $\beta$ & $P$ & $B$ & $P$ & $\beta$ & $P$ \\
\hline Episodic memory & -0.049 & 0.42 & 0.095 & 0.28 & -0.03 & 0.62 & -0.089 & 0.26 \\
\hline Semantic memory & -0.035 & 0.61 & 0.097 & 0.34 & 0.014 & 0.84 & 0.031 & 0.59 \\
\hline Block-design & 0.023 & 0.71 & 0.029 & 0.75 & -0.037 & 0.61 & -0.107 & 0.24 \\
\hline Two-back test & 0.14 & 0.19 & -0.245 & 0.019 & -0.028 & 0.66 & -0.096 & 0.23 \\
\hline
\end{tabular}

Two models were tested: A included age, gender, and level of education as covariates; B included the same covariates plus the interaction term cortisol measure $\times$ gender. The 2-back test had a significant AUC cortisol x gender interaction and, therefore, results were presented from model B.

prefrontal cortex and cortisol exposure in humans, without a clear link between HC and cortisol levels.

Studies testing the association between cortisol levels, cortical surface area, and thickness, instead of the composite measure cortical volume, are sparse. This is important since recent findings indicate that cortical surface area and thickness are genetically uncorrelated (27), and seem to be differentially related to cognitive performance (27) and diseases such as Alzheimer dementia (28). Based on findings among 388 middle-aged men, we hypothesized that higher cortisol levels would be related to a thinner cortex but not smaller cortical surface area (15). However, our results instead suggest a link between high total exposure and small cortical surface area, but not thin cortex in several prefrontal brain regions.

In our sample, the negative association between AUC for cortisol levels and cortical surface area was particularly strong in the left caudal anterior cingulate gyrus. This brain region has previously been suggested to be important for regulation of the HPAA; elderly men with impaired cortisol suppression after dexamethasone have smaller left anterior cingulate cortex volumes than those with normal suppression (29). Our result extends this finding to include both genders and cortisol levels under physiological conditions as compared with levels after pharmacologic suppression. We expected a weaker association between cortisol levels and brain morphology in women than men, based on experimental data $(18,30)$, but this was not supported by our results. Importantly, all women in our sample were older than 55 years and thus were probably postmenopausal. This may have had a major influence in our results, and further studies including both premenopausal and postmenopausal women are warranted.

In rodents, lesions in the medial PFC increase cortisol levels in response to stressors (31). Furthermore, chronic stress causes a loss of dendritic spines in the rat medial PFC, subsequently leading to increased HPAA response to an acute stressors (8). Taken together, the relationship between the medial PFC and the HPAA seems to be bidirectional: the medial PFC inhibits the HPAA but is adversely affected by increased stress and glucocorticoid levels. Our results suggest that the medial prefrontal cortex is linked to the HPAA in humans as well. With our study design, we cannot test a cause-effect relationship between cortisol levels and medial PFC structure. However, chronic glucocorticoid treatment and Cushing's syndrome with chronically elevated cortisol levels are associated with lower prefrontal brain volume, altered functional connectivity of the limbic system, and impaired cognitive functions $(32,33,34,35)$, suggesting that elevated cortisol levels cause, rather than are a consequence of, altered PFC structure and function.

We did not find any convincing direct associations between cortisol levels and the tested cognitive domains. This is in contrast to earlier data showing associations between elevated cortisol levels in healthy individuals and impairment of cognitive functions dependent on the PFC such as executive functions, attention, processing speed, and semantic memory $(11,12,16,36)$. However, the positive relationship between surface area of the left anterior cingulate gyrus and semantic memory performance as well as visuospatial performance may indicate a potential for high cortisol levels to cause impaired cognition. The clinical importance of an association between cortisol levels and brain structure but not cognitive functions is not clear. Future prospective studies linking cortisol levels to cognitive decline and alterations in brain structure over time could shed further light on a putative negative effect of increased cortisol levels on cognitive functions.

The lack of association between either total cortisol exposure or late night cortisol levels and volume of the HC do not support data from rodents and primates, suggesting powerful effects of increased glucocorticoid levels on HC morphology (37, 38, 39, 40). However, these experimental studies have used high doses of glucocorticoids or stress exposure while the majority of participants in our study had cortisol levels within the 
normal range. Prospectively increasing cortisol levels have been related to reduced HC volume in elderly men (9), but other studies of HPAA activity have not confirmed these findings $(15,16,41)$. Furthermore, a cross-sectional analysis of subjects with atherosclerotic disease revealed that high late-evening salivary cortisol levels and high morning post-dexamethasone cortisol levels were related to lower HC volume (14). In summary, the association between cortisol levels and HC volume appears to be weak and perhaps may only be evident when examined prospectively or in individuals with other comorbidities. Notably, a potential link between high cortisol levels and hippocampal atrophy may be masked when measuring total HC volume since experimental studies suggests that subregions within the $\mathrm{HC}$ such as the dentate gyrus may be particularly sensitive to glucocorticoids $(2,42)$. Furthermore, repeated cortisol measurements over several days would provide a better measure of chronic cortisol exposure. Thus, future studies using high-resolution MRI to measure volume of $\mathrm{HC}$ subregions as well as chronic cortisol exposure would be of great interest.

Potential limitations in our study include the singleday cortisol measurements. Importantly, basal serum cortisol levels measured more than 1 year apart have a correlation coefficient of $\sim 0.5(43,44)$, a correlation that seems to be consistent also after exposure to stressors (44). Diurnal rhythm may have influenced cortisol levels, and we lacked information about the subjects' waking times, the exact sampling time, and whether cortisol was sampled during a workday or weekend. Other factors that may influence cortisol secretion, e.g. physical activity was not controlled for either. Due to logistic purposes, the cortisol samples were collected several months before the MRI examination. Morphological brain changes could, therefore, have occurred after the saliva sampling. However, all PFC regions that were associated with cortisol levels in our study have been found to be associated with indices of HPAA activity or to be affected by elevated cortisol levels previously $(15,29,32,34,45)$.

Strengths of the study include the use of a large population-based sample of both women and men with a relatively broad age span. Detailed medical histories enabled us to control for possible influences of neurological, psychiatric, cardiovascular, and metabolic diseases, increasing the external validity of our results compared with previous studies. Furthermore, we corrected our results for multiple comparisons, in contrast to previous studies, which have not used any means of correction.

In conclusion, in a sample of middle-aged and elderly women and men drawn from a representative population cohort, we found an inverse association between cortisol levels and medial prefrontal cortex morphology in particular. Future prospective studies could yield further insight into the suggested cause-effect relationship between cortisol levels, brain structure, and cognitive functions.

Supplementary data

This is linked to the online version of the paper at http://dx.doi.org/10.1530/ EJE-16-0352.

Declaration of interest

The authors report no conflict of interest.

\section{Funding}

The Betula Prospective Cohort study of Memory and Aging is supported by a Knut and Alice Wallenberg Scholar grant to $L \mathrm{~N}$.

\section{Acknowledgments}

The authors thank the staff of the Betula project and Micael Andersson.

\section{References}

1 McEwen BS. Protection and damage from acute and chronic stress: allostasis and allostatic overload and relevance to the pathophysiology of psychiatric disorders. Annals of the New York Academy of Sciences 20041032 1-7. (doi:10.1196/annals.1314.001)

2 Seckl JR, Dickson KL, Yates C \& Fink G. Distribution of glucocorticoid and mineralocorticoid receptor messenger RNA expression in human postmortem hippocampus. Brain Research $1991561332-337$. (doi:10.1016/0006-8993(91)91612-5)

3 Berardelli R, Karamouzis I, D’Angelo V, Zichi C, Fussotto B, Giordano R, Ghigo E \& Arvat E. Role of mineralocorticoid receptors on the hypothalamus-pituitary-adrenal axis in humans. Endocrine 201343 51-58. (doi:10.1007/s12020-012-9750-8)

4 Ratka A, Sutanto W, Bloemers M \& de Kloet ER. On the role of brain mineralocorticoid (type I) and glucocorticoid (type II) receptors in neuroendocrine regulation. Neuroendocrinology 198950 117-123.

5 Cabeza R \& Nyberg L. Imaging cognition II: an empirical review of 275 PET and fMRI studies. Journal of Cognitive Neuroscience 200012 1-47. (doi:10.1162/08989290051137585)

6 Seckl JR \& Olsson T. Glucocorticoid hypersecretion and the ageimpaired hippocampus: cause or effect? Journal of Endocrinology 1995 145 201-211. (doi:10.1677/joe.0.1450201)

7 Radley JJ \& Morrison JH. Repeated stress and structural plasticity in the brain. Ageing Research Reviews 20054 271-287. (doi:10.1016/j. arr.2005.03.004)

8 Radley JJ, Anderson RM, Hamilton BA, Alcock JA \& Romig-Martin SA. Chronic stress-induced alterations of dendritic spine subtypes predict functional decrements in an hypothalamo-pituitaryadrenal-inhibitory prefrontal circuit. Journal of Neuroscience 201333 14379-14391. (doi:10.1523/JNEUROSCI.0287-13.2013)

9 Lupien SJ, de Leon M, de Santi S, Convit A, Tarshish C, Nair NP, Thakur M, McEwen BS, Hauger RL \& Meaney MJ. Cortisol levels during human aging predict hippocampal atrophy and memory deficits. Nature Neuroscience 19981 69-73. (doi:10.1038/271)

10 Lee BK, Glass TA, McAtee MJ, Wand GS, Bandeen-Roche K, Bolla KI \& Schwartz BS. Associations of salivary cortisol with cognitive function 
in the Baltimore memory study. Archives of General Psychiatry 200764 810-818. (doi:10.1001/archpsyc.64.7.810)

11 Franz CE, O’Brien RC, Hauger RL, Mendoza SP, Panizzon MS, Prom-Wormley E, Eaves LJ, Jacobson K, Lyons MJ, Lupien S et al. Cross-sectional and 35-year longitudinal assessment of salivary cortisol and cognitive functioning: the Vietnam Era twin study of aging. Psychoneuroendocrinology 201136 1040-1052. (doi:10.1016/j. psyneuen.2011.01.002)

12 Beluche I, Carriere I, Ritchie K \& Ancelin ML. A prospective study of diurnal cortisol and cognitive function in communitydwelling elderly people. Psychological Medicine 201040 1039-1049. (doi:10.1017/S0033291709991103)

13 O’Brien JT, Lloyd A, McKeith I, Gholkar A \& Ferrier N. A longitudinal study of hippocampal volume, cortisol levels, and cognition in older depressed subjects. American Journal of Psychiatry 2004161 2081-2090.

14 Knoops AJ, Gerritsen L, van der Graaf Y, Mali WP \& Geerlings MI. Basal hypothalamic pituitary adrenal axis activity and hippocampal volumes: the SMART-Medea study. Biological Psychiatry 201067 1191-1198. (doi:10.1016/j.biopsych.2010.01.025)

15 Kremen WS, O’Brien RC, Panizzon MS, Prom-Wormley E, Eaves LJ, Eisen SA, Eyler LT, Hauger RL, Fennema-Notestine C, Fischl B et al. Salivary cortisol and prefrontal cortical thickness in middle-aged men: a twin study. Neuroimage 201053 1093-1102. (doi:10.1016/j. neuroimage.2010.02.026)

16 MacLullich AM, Deary IJ, Starr JM, Ferguson KJ, Wardlaw JM \& Seckl JR. Plasma cortisol levels, brain volumes and cognition in healthy elderly men. Psychoneuroendocrinology 200530 505-515. (doi:10.1016/j.psyneuen.2004.12.005)

17 Galea LA, McEwen BS, Tanapat P, Deak T, Spencer RL \& Dhabhar FS. Sex differences in dendritic atrophy of CA3 pyramidal neurons in response to chronic restraint stress. Neuroscience 199781 689-697. (doi:10.1016/S0306-4522(97)00233-9)

18 Garrett JE \& Wellman CL. Chronic stress effects on dendritic morphology in medial prefrontal cortex: sex differences and estrogen dependence. Neuroscience 2009162 195-207. (doi:10.1016/j. neuroscience.2009.04.057)

19 Nilsson LG, Backman L, Erngrund K, Nyberg L, Adolfsson R, Bucht G, Karlsson S, Widing M \& Winblad B. The Betula prospective cohort study: Memory, health and aging. Aging Neuropsychology and Cognition 19974 1-32. (doi:10.1080/13825589708256633)

20 Crum RM, Anthony JC, Bassett SS \& Folstein MF. Population-based norms for the Mini-Mental State Examination by age and educational level. JAMA 1993269 2386-2391.

21 Pruessner JC, Kirschbaum C, Meinlschmid G \& Hellhammer DH. Two formulas for computation of the area under the curve represent measures of total hormone concentration versus time-dependent change. Psychoneuroendocrinology 200328 916-931. (doi:10.1016/ S0306-4530(02)00108-7)

22 Fischl B, Salat DH, Busa E, Albert M, Dieterich M, Haselgrove C, van der Kouwe A, Killiany R, Kennedy D, Klaveness S et al. Whole brain segmentation: automated labeling of neuroanatomical structures in the human brain. Neuron 200233 341-355. (doi:10.1016/S08966273(02)00569-X)

23 Desikan RS, Segonne F, Fischl B, Quinn BT, Dickerson BC, Blacker D, Buckner RL, Dale AM, Maguire RP, Hyman BT et al. An automated labeling system for subdividing the human cerebral cortex on MRI scans into gyral based regions of interest. Neuroimage $2006 \mathbf{3 1}$ 968-980. (doi:10.1016/j.neuroimage.2006.01.021)

24 Buckner RL, Head D, Parker J, Fotenos AF, Marcus D, Morris JC \& Snyder AZ. A unified approach for morphometric and functional data analysis in young, old, and demented adults using automated atlas-based head size normalization: reliability and validation against manual measurement of total intracranial volume. Neuroimage 200423 724-738. (doi:10.1016/j. neuroimage.2004.06.018)
25 Cerqueira JJ, Almeida OF \& Sousa N. The stressed prefrontal cortex. Left? Right! Brain, Behavior and Immunity 200822 630-638. (doi:10.1016/j.bbi.2008.01.005)

26 Benjamini Y \& Hochberg Y. Controlling the false discovery rate: a practical and powerful approach to multiple testing. Journal of the Royal Statistical Society. Series B (Methodological) 199557 289-300.

27 Vuoksimaa E, Panizzon MS, Chen CH, Fiecas M, Eyler LT, FennemaNotestine C, Hagler DJ, Fischl B, Franz CE, Jak A et al. The genetic association between neocortical volume and general cognitive ability is driven by global surface area rather than thickness. Cerebral Cortex 201525 2127-2137. (doi:10.1093/cercor/bhu018)

28 Dickerson BC, Feczko E, Augustinack JC, Pacheco J, Morris JC, Fischl B \& Buckner RL. Differential effects of aging and Alzheimer's disease on medial temporal lobe cortical thickness and surface area. Neurobiology of Aging 200930 432-440. (doi:10.1016/j. neurobiolaging.2007.07.022)

29 MacLullich AM, Ferguson KJ, Wardlaw JM, Starr JM, Deary IJ \& Seckl JR. Smaller left anterior cingulate cortex volumes are associated with impaired hypothalamic-pituitary-adrenal axis regulation in healthy elderly men. Journal of Clinical Endocrinology \& Metabolism 200691 1591-1594. (doi:10.1210/jc.2005-2610)

30 Shansky RM, Hamo C, Hof PR, Lou W, McEwen BS \& Morrison JH. Estrogen promotes stress sensitivity in a prefrontal cortex-amygdala pathway. Cerebral Cortex 201020 2560-2567. (doi:10.1093/cercor/ bhq003)

31 Diorio D, Viau V \& Meaney MJ. The role of the medial prefrontal cortex (cingulate gyrus) in the regulation of hypothalamic-pituitaryadrenal responses to stress. Journal of Neuroscience 199313 3839-3847.

32 Andela CD, van der Werff SJ, Pannekoek JN, van den Berg SM, Meijer OC, van Buchem MA, Rombouts SA, van der Mast RC, Romijn JA, Tiemensma J et al. Smaller grey matter volumes in the anterior cingulate cortex and greater cerebellar volumes in patients with long-term remission of Cushing's disease: a case-control study. European Journal of Endocrinology 2013169 811-819. (doi:10.1530/EJE-13-0471)

33 Brown ES, Woolston DJ, Frol A, Bobadilla L, Khan DA, Hanczyc M, Rush AJ, Fleckenstein J, Babcock E \& Cullum CM. Hippocampal volume, spectroscopy, cognition, and mood in patients receiving corticosteroid therapy. Biological Psychiatry 200455 538-545. (doi:10.1016/j.biopsych.2003.09.010)

34 van der Werff SJ, Pannekoek JN, Andela CD, Meijer OC, van Buchem MA, Rombouts SA, van der Mast RC, Biermasz NR, Pereira AM $\&$ van der Wee NJ. Resting-state functional connectivity in patients with long-term remission of Cushing's disease. Neuropsychopharmacology 2015 40 1888-1898. (doi:10.1038/npp.2015.38)

35 Ragnarsson O, Berglund P, Eder DN \& Johannsson G. Long-term cognitive impairments and attentional deficits in patients with Cushing's disease and cortisol-producing adrenal adenoma in remission. Journal of Clinical Endocrinology \& Metabolism 201297 E1640-E1648. (doi:10.1210/jc.2012-1945)

36 Lupien S, Lecours AR, Lussier I, Schwartz G, Nair NP \& Meaney MJ Basal cortisol levels and cognitive deficits in human aging. Journal of Neuroscience 199414 2893-2903.

37 Sapolsky RM, Uno H, Rebert CS \& Finch CE. Hippocampal damage associated with prolonged glucocorticoid exposure in primates. Journal of Neuroscience 199010 2897-2902.

38 Gould E, McEwen BS, Tanapat P, Galea LA \& Fuchs E. Neurogenesis in the dentate gyrus of the adult tree shrew is regulated by psychosocial stress and NMDA receptor activation. Journal of Neuroscience 199717 2492-2498.

39 Magarinos AM, McEwen BS, Flugge G \& Fuchs E. Chronic psychosocial stress causes apical dendritic atrophy of hippocampal CA3 pyramidal neurons in subordinate tree shrews. Journal of Neuroscience 199616 3534-3540.

40 Magarinos AM \& McEwen BS. Stress-induced atrophy of apical dendrites of hippocampal CA3c neurons: involvement of 
glucocorticoid secretion and excitatory amino acid receptors. Neuroscience 199569 89-98. (doi:10.1016/0306-4522(95)00259-L)

41 Gold SM, Dziobek I, Rogers K, Bayoumy A, McHugh PF \& Convit A. Hypertension and hypothalamo-pituitary-adrenal axis hyperactivity affect frontal lobe integrity. Journal of Clinical Endocrinology \& Metabolism 200590 3262-3267. (doi:10.1210/jc.2004-2181)

42 Sapolsky RM, Krey LC \& McEwen BS. The neuroendocrinology of stress and aging: the glucocorticoid cascade hypothesis. Endocrine Reviews 19867 284-301. (doi:10.1210/edrv-7-3-284)

43 Huizenga NA, Koper JW, de Lange P, Pols HA, Stolk RP, Grobbee DE, de Jong FH \& Lamberts SW. Interperson variability but intraperson stability of baseline plasma cortisol concentrations, and its relation to feedback sensitivity of the hypothalamo-pituitary-adrenal axis to a low dose of dexamethasone in elderly individuals. Journal of Clinical Endocrinology \& Metabolism 199883 47-54. (doi:10.1210/ jcem.83.1.4498)

44 Burleson MH, Poehlmann KM, Hawkley LC, Ernst JM, Berntson GG, Malarkey WB, Kiecolt-Glaser JK, Glaser R \& Cacioppo JT.

Neuroendocrine and cardiovascular reactivity to stress in mid-aged and older women: long-term temporal consistency of individual differences. Psychophysiology 200340 358-369. (doi:10.1111/ psyp.2003.40.issue-3)

45 Boehringer A, Tost H, Haddad L, Lederbogen F, Wust S, Schwarz E \& Meyer-Lindenberg A. Neural correlates of the cortisol awakening response in humans. Neuropsychopharmacology 201540 2278-2285. (doi:10.1038/npp.2015.77)

Received 20 April 2016

Revised version received 11 May 2016

Accepted 17 May 2016 